OPEN ACCESS

Edited by:

Shixiu Wu,

Hangzhou Cancer Hospital, China

Reviewed by:

Suyan Bi,

Chinese Academy of Medical Sciences and Peking Union

Medical College, China Carlo Greco,

Policlinico Universitario Campus Bio-Medico, Italy

*Correspondence: Mengchao Zhang

zhangmengchao@jlu.edu.cn

${ }^{t}$ These authors have contributed equally to this work

Specialty section:

This article was submitted to Gastrointestinal Cancers,

a section of the journal

Frontiers in Oncology

Received: 19 April 2021 Accepted: 21 June 2021 Published: 07 July 2021

Citation:

Li Z, Dai H, Liu Y, Pan F, Yang $Y$ and Zhang $M$ (2021)

Radiomics Analysis of MultiSequence MR Images For Predicting

Microsatellite Instability Status Preoperatively in Rectal Cancer.

Front. Oncol. 11:697497. doi: 10.3389/fonc.2021.697497

\section{Radiomics Analysis of Multi- Sequence MR Images For Predicting Microsatellite Instability Status Preoperatively in Rectal Cancer}

\author{
Zongbao $\mathrm{Li}^{1+}$, Hui Dai ${ }^{2+}$, Yunxia Liu ${ }^{1}$, Feng Pan ${ }^{1}$, Yanyan Yang ${ }^{1}$ and Mengchao Zhang ${ }^{1 *}$ \\ ${ }^{1}$ China-Japan Union Hospital of Jilin University, Changchun, China, 2 The First Affiliated Hospital of Soochow University, \\ Suzhou, China
}

Background: Immunotherapy, adjuvant chemotherapy, and prognosis of colorectal cancer are associated with MSI. Biopsy pathology cannot fully reflect the MSI status and heterogeneity of rectal cancer.

Purpose: To develop a radiomic-based model to preoperatively predict MSI status in rectal cancer on MRI.

Assessment: The patients were divided into two cohorts (training and testing) at a 7:3 ratio. Radiomics features, including intensity, texture, and shape, were extracted from the segmented volumes of interest based on T2-weighted and ADC imaging.

Statistical Tests: Independent sample t test, Mann-Whitney test, the chi-squared test, Receiver operating characteristic curves, calibration curves, decision curve analysis and multi-variate logistic regression analysis

Results: The radiomics models were significantly associated with MSI status. The T2based model showed an area under the curve of 0.870 with $95 \% \mathrm{Cl}: 0.794-0.945$ (accuracy, 0.845; specificity, 0.714; sensitivity, 0.976) in training set and 0.895 with $95 \%$ $\mathrm{Cl}, 0.777-1.000$ (accuracy, 0.778; specificity, 0.887; sensitivity, 0.772) in testing set. The ADC-based model had an AUC of 0.790 with 95\% Cl: 0.794-0.945 (accuracy, 0.774; specificity, 0.714; sensitivity, 0.976) in training set and 0.796 with $95 \% \mathrm{Cl}, 0.777-1.000$ (accuracy, 0.778; specificity, 0.889; sensitivity, 0.772) in testing set. The combined model integrating T2 and ADC features showed an AUC of 0.908 with $95 \% \mathrm{Cl}: 0.845-0.971$ (accuracy, 0.857; specificity, 0.762; sensitivity, 0.952) in training set and 0.926 with $95 \%$ $\mathrm{Cl}$ : 0.813-1.000 (accuracy, 0.852; specificity, 1.000; sensitivity, 0.778) in testing set. Calibration curve showed that the combined score had a good calibration degree, and the decision curve demonstrated that the combined score was of benefit for clinical use.

Data Conclusion: Radiomics analysis of T2W and ADC images showed significant relevance in the prediction of microsatellite status, and the accuracy of combined model of ADC and T2W features was better than either alone.

Keywords: magnetic resonance, rectal cancer, microsatellite instability, radiomics, multi-sequence MR 


\section{INTRODUCTION}

The incidence rate of rectal cancer patients was the third and the mortality rate was the fourth in malignant tumors (1). The 5 -year disease-free survival rate of colorectal cancer was associated with MIS status (2). Colorectal cancer patients with the MSI has a better prognosis than with MSS, meaning that MSI status can be used as a good prognostic indicator $(3,4)$. Patients with MSI only benefit from the 5-FU-based adjuvant chemotherapy, but the rectal cancer with MSS does not respond to 5-FU-based neoadjuvant therapy (5-7). Some patients with dDDM/MSI tumor respond to immune checkpoint inhibitors (ICI) therapy (8). Therefore, the microsatellite status of colorectal cancer is helpful to the selection of neoadjuvant therapy and predicts the prognosis of colorectal cancer. The status of MSI is assessed using colonoscopy biopsy. However, this method had two challenges. First, the DNA extracted from the sample obtaining from colonoscopy biopsy may not meet the minimum quality criteria from the genetic assay; second, because of the high heterogeneity of colorectal cancer, the accurate MSI status of colorectal cancer cannot be obtained by puncture pathology examination (9). Therefore, we need to find a way to preoperatively predict MSI status and reflect heterogeneity of rectal cancer.

Now, there is a certain correlation between the texture parameters extracted from tumor images and the gene expression of tumors. CT image texture parameters of colorectal cancer have certain correlation with KRAS/NRAS/ BRAF gene mutations in colorectal cancer, and the texture parameters extracted from MRI images can help predict TCGA/TCIA molecular subtypes in breast cancer $(10,11)$. We hypothesize that the MR imaging radiomics parameter modeling can predict the MSI statue of rectal cancer. Therefore, the purpose of this study is to assess the performance of predicting the rectal cancer MSI status used MR radiomics.

\section{MATERIALS AND METHODS}

\section{Patients}

This prospective study was approved by the institutional ethical committee of our hospital, and all patients signed a written informed consent prior to MR. From January 2016 to February 2019, 90 patients who met the following criteria were included in our study. Inclusion criteria: (1) patients who were highly suspected of rectal cancer underwent colonoscopy and confirmed by postoperative pathology; (2) IHC examination was performed to determine the expression status of rectal cancer mismatch repair protein. Exclusion criteria: (1) the patients have severe systemic disease and MR examination contraindications. (2) The artifacts of rectal cancer MR lesions

\footnotetext{
Abbreviations: MR, magnetic resonance; MSI, microsatellite instability; MSS, microsatellite stable; ROI, region of interest; AUC, area under the curve; ICC, intraclass correlation coefficient; SMOTE, synthetic minority oversampling technique; ROC, receiver operating characteristic; DCE-MRI, dynamic contrastenhanced magnetic resonance imaging; MVD, micro-vessel density.
}

were heavy, and the lesions were incomplete. (3) Without the result of expression of mismatch repair protein. The details of the patient selection are shown in Figure 1.

\section{Determination of MSI Statues}

Specimens were fixed with $4 \%$ formaldehyde, embedded in paraffin, serially sectioned at $4 \mu \mathrm{m}$, and stained with conventional HE. Immunohistochemical staining was performed using the EnVision two-step method. The primary antibody was rabbit anti-human MLH1, MSH2, MSH6, PMS2. DAB coloration, hematoxylin counterstaining. Nuclear staining of tumor tissue stromal cells and normal non-tumor mucosa was used as a positive control; antibody diluent was used instead of an antibody as a negative control. In the area where the nuclear staining is good in the internal control (normal intestinal glandular epithelium and interstitial cells, etc.), the tumor cell nuclear staining is positive, and the coloration is negative, and if the protein expression is negative, it is assumed that there is loss of mismatch repair protein expression, meaning that the tumor is MSI.

\section{MR Image Acquisition, Preprocessing, and Segmentation}

Standardized MR scans were performed with a $3.0 \mathrm{~T}$ scanner (Siemens skyra3.0T, Germany): all the patients underwent routine MR and multiple b-value DW sequence scans. The position of the tumor was determined by sagittal T2W-TSE sequence image, and the $\mathrm{T} 2 \mathrm{~W}$ image perpendicular to the rectal axis (TR/TE, 3380/95 ms; field of view [FOV], $210 \mathrm{~mm} \times$ $210 \mathrm{~mm}$; voxel size, $0.7 \mathrm{~mm} \times 0.7 \mathrm{~mm} \times 3.0 \mathrm{~mm}$ ), DW sequence image (voxel size, $2.4 \mathrm{~mm} \times 2.4 \mathrm{~mm} \times 3.0 \mathrm{~mm}$; b values, 50,800 ) and $\mathrm{ADC}$ image were automatically generated.

All the T2W and ADC images were uploaded to A.K software (Artificial Intelligence Kit; A.K., GE Healthcare, China) for histogram matching and homogenization.

Two radiologists with experience of 15 and 30 years used the open-source ITK-SNAP software (version 3.6.0 Apr 1, 2017, Copyright(c) 1998-2017 Paul A. Yushkevich Guido Gerig) to manually delineated the ROI of the tumor in the T2W image and the ADC image with the patient's colonoscopy results, they sketch the lesions layer by layer from the edge of the tumor independently, and then generated the 3D-ROI (Figure 2).

\section{Radiomics Features Extraction}

All images and ROIs were uploaded to AK software and then 385 features were automatically calculated, as shown in Figure 3.

\section{Features Selection and Modeling}

First, in order to assess the intra-observer reproducibility of the radiomics features so as to establish a robust model, we calculated the intra-class coefficient correlation (ICC) index. The features of ICC $\geq 0.75$ were retained, which yielded a relatively high intra-observer stability in the segmented tumor volume, and the features of ICC $<0.75$ were abandoned.

Then, the remaining stable features were subjected to random forest model and the top 5 of important index were retained, and the rest were discarded. Finally, multivariate logic stepwise regression was used to remove meaningless features backward 
Rectal cancer underwent MR examination

Confirmed the expression of mismatch repair protein by immunohistochemistry

From January 2016 to February 2019 (N=165)

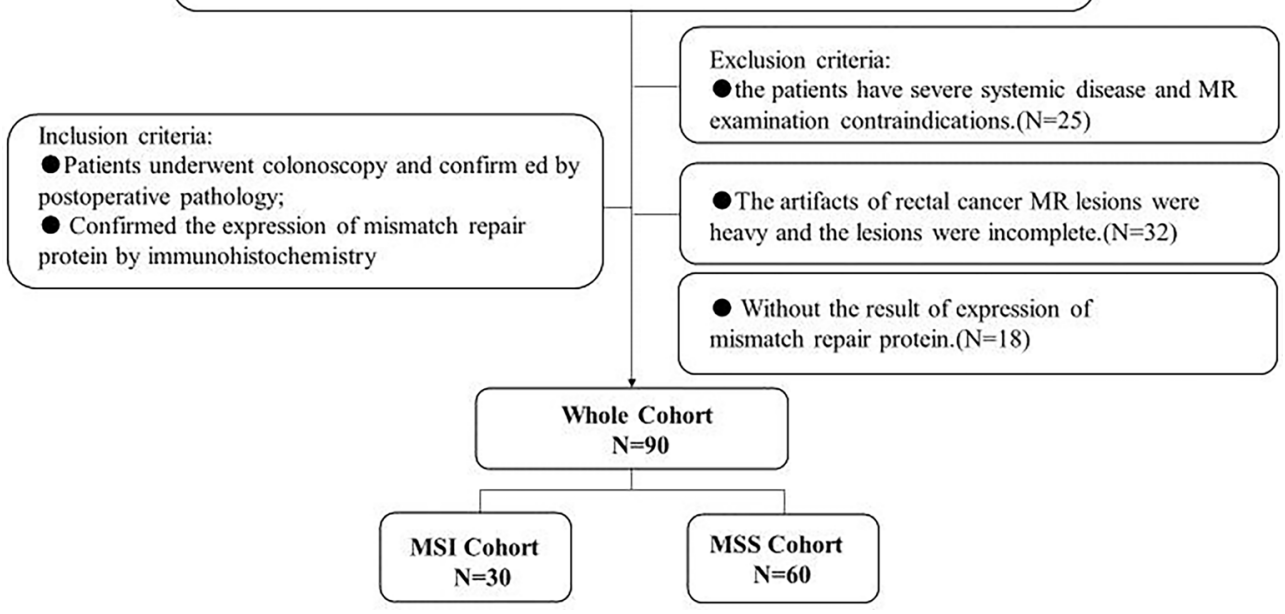

FIGURE 1 | Flow diagram of patient selection.

and construct a radiomics score, which called ADC score and T2 score.

To generate an integrated model, ADC score and T2 score were combined using multivariate logistic regression analysis, after which, a combined score was constructed.

\section{Balancing Data Samples}

We randomly divided the proportion of these 90 patients into 7:3 into training and testing sets. Considering that logistic regression performs best when case-to-noncase ratio is 1:1, the number of patients with MSI was smaller than the number of patients with MSS, and the sample imbalance would have an adverse impact on the performance of a classifier, Thus, we used the synthetic minority oversampling technique (SMOTE) (12) in the training set to increase the weight of minority samples and to balance the samples of patients, where the ratio of MSI and MSS was 1:1. However, we did not apply the SMOTE in testing set mainly because: firstly the bias data set was independent from the
A

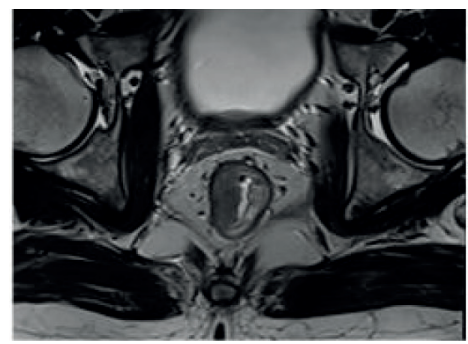

D

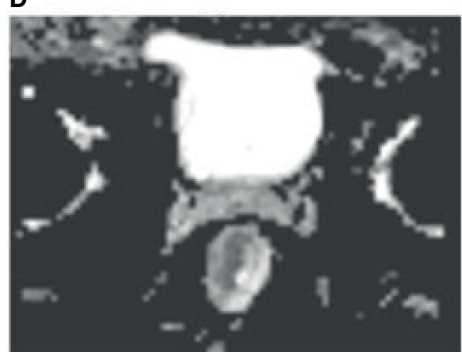

B

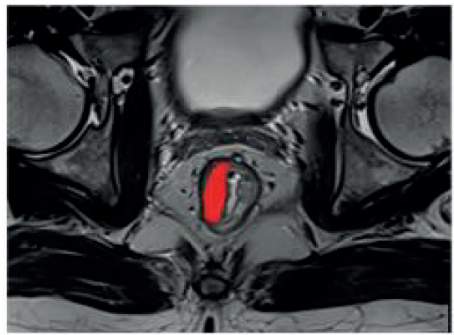

E

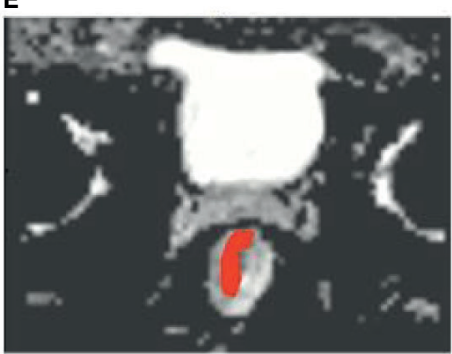

C
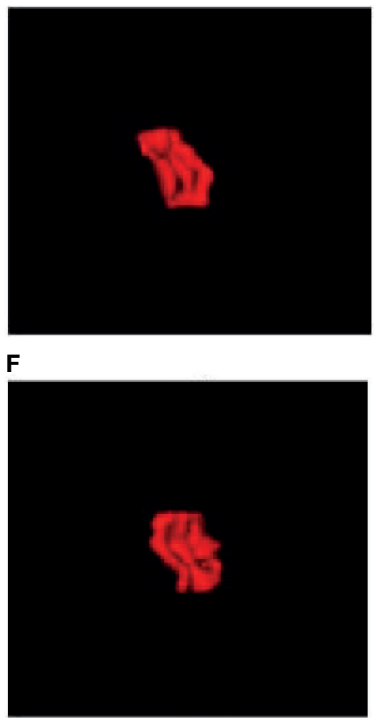

FIGURE 2 | Examples of rectal cancer segmentation. (A-C) T2WI images, (D-F) ADC images, (C, F) 3D view. 


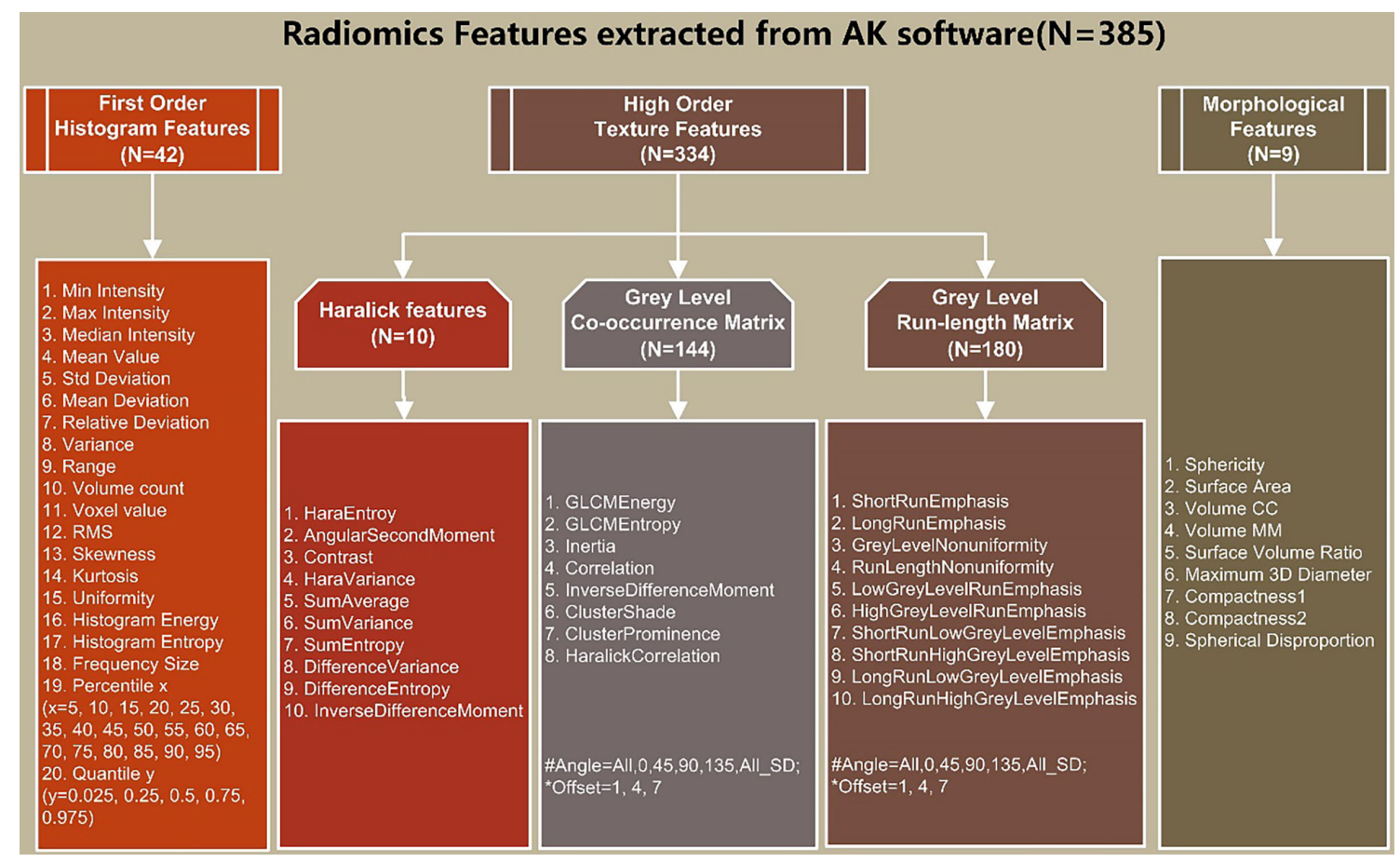

FIGURE 3 | Details of extracted radiomics features: (1) First-order histogram features $(n=42)$; (2) high-order texture features $(n=334)$ : Haralick features ( $n=10)$, gray level co-occurrence matrix (GLCM) ( $n=144)$, and gray-level run-length matrix (GLRLM) $(n=180)$; (3) morphological features $(n=9)$.

training set, so it had no effect at modeling, and second, the incidence of MSI was lower than MSS in the real world, so we use the real testing set to obtain a real testing result of the model. In the end, we randomly selected one tenth of the patients with MSI and patients with MSS to test the model.

\section{Statistical Analysis}

All the statistical analyses were performed with $\mathrm{R}$ language (Version 1.0.143- ${ }^{{ }}$2009-2016 R Studio, Inc.). The differences in patient features between patients with MSI and MSS were assessed by the independent-sample $t$ test or Mann-Whitney test according to the data distribution type. The chi-squared test was used to compare the significance of the differences between categorical variables. Receiver operating characteristic (ROC) curves were plotted to evaluate the diagnostic performance of the radiomics score in both training and testing sets. Area under the ROC curve (AUC) with 95\% confidence interval (CI), specificity, sensitivity, and accuracy were calculated. DeLong test was used to compare the differences of AUC values between different models in the training and testing set. To evaluate whether the models were well-calibrated or not, calibration curves were plotted in both training and testing sets. Decision curve analysis (DCA) was conducted to determine the clinical usefulness of the models by quantifying the net benefits at different threshold probabilities in both training and testing sets. The flowchart of our study was shown in Figure 4.

\section{RESULTS}

\section{Clinical Characteristics}

In our research, 60 patients were MSS (38 males, 22 females; mean age, 61.12; age range, $31-82$ years) and 30 patients were MSI (16 males, 14 females, average age, 60.29 years; age range, 30-83 years. Our results showed that there was no significant difference in clinical features between MSI and MSS patients with age, gender, tumor location, size, differentiation degree, TNM stage, hypertension, diabetes, family history of cancer, and smoking and alcohol history. The details of baseline characteristics results were shown in Table $\mathbf{1}$.

\section{Feature Selection and Radiomics Models Building}

After consistency analysis, a total of 203 and 89 radiomics features, respectively, extracted from $\mathrm{T} 2 \mathrm{~W}$ and $\mathrm{ADC}$ images had ICC values of more than 0.75 and were then used for features selection and modeling.

Then top 5 features based on T2W and ADC imaging, which had the biggest mean decrease accuracy value in the random forest model, were obtained. According to their importance, the features were ranked as shown in Figure 5.

The five selected features were then entered into multivariate logistic regression using backward elimination method, after which, a total of two and three features were retained respectively from the $\mathrm{T} 2 \mathrm{~W}$ and $\mathrm{ADC}$ images for the final modeling. Radiomics scores (Rad-scores) were calculated for 


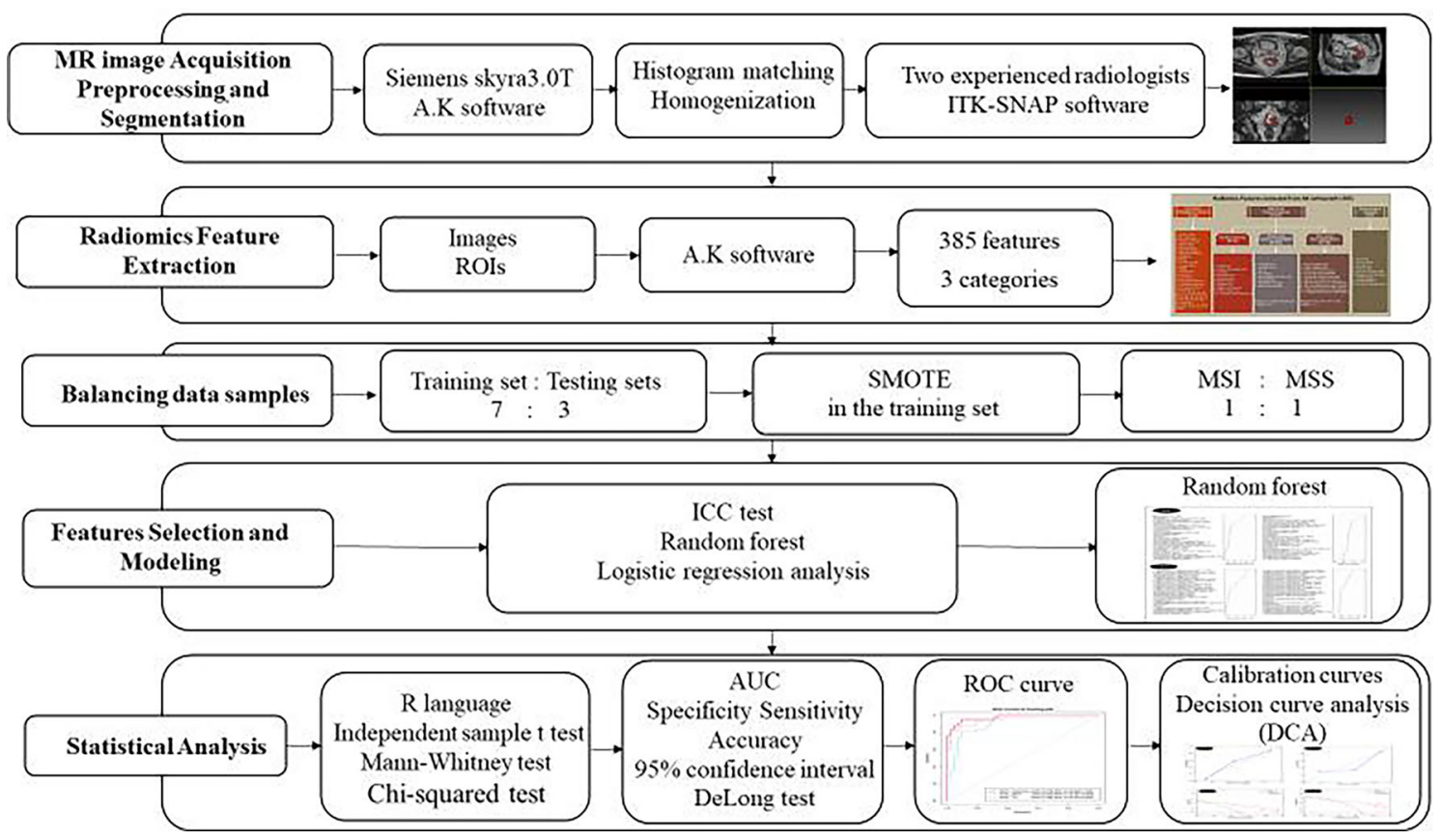

FIGURE 4 | Flow diagram of our study.

TABLE 1 | The results of baseline characteristics.

\begin{tabular}{|c|c|c|c|c|}
\hline Characteristic & & MSS (N=60) & MSI (N=30) & $P$ values \\
\hline Age, mean \pm SD (years) & & $61.03 \pm 11.23$ & $58.05 \pm 10.55$ & 0.252 \\
\hline \multirow[t]{2}{*}{ Gender, n (\%) } & Male & 38(63.3) & $15(50.0)$ & 0.226 \\
\hline & Female & $22(36.7)$ & $15(50.0)$ & \\
\hline \multirow[t]{3}{*}{ Tumor location, n (\%) } & High & $8(13.3)$ & $4(13.3)$ & 0.983 \\
\hline & Medium & $39(65.0)$ & 19(63.3) & \\
\hline & Low & $13(21.7)$ & $7(23.4)$ & \\
\hline Length of tumor, mean \pm SD $(\mathrm{cm})$ & & $3.97 \pm 1.81$ & $4.46 \pm 1.16$ & 0.334 \\
\hline \multirow[t]{4}{*}{ Differentiation, n (\%) } & Well & $3(5.0)$ & $O(0.0)$ & 0.159 \\
\hline & Moderately & $43(71.7)$ & $17(56.7)$ & \\
\hline & Poorly & $10(16.7)$ & $8(26.7)$ & \\
\hline & Mucinous & $4(6.6)$ & $5(16.6)$ & \\
\hline \multirow[t]{4}{*}{ Stages, n (\%) } & $\mathbf{I}$ & $22(36.7)$ & $8(26.7)$ & 0.606 \\
\hline & II & 14(23.3) & 9(30.0) & \\
\hline & III & $24(40.0)$ & 13(43.3) & \\
\hline & IV & $0(0.0)$ & $\mathrm{O}(0.0)$ & \\
\hline \multirow[t]{4}{*}{ T-staging, n (\%) } & T1 & $4(6.7)$ & $\mathrm{O}(0.0)$ & 0.434 \\
\hline & T2 & 23(38.3) & 10(33.3) & \\
\hline & T3 & $32(53.3)$ & 19(63.3) & \\
\hline & T4 & $1(1.7)$ & $1(3.4)$ & \\
\hline \multirow[t]{2}{*}{$\mathrm{N}$-staging, $\mathrm{n}(\%)$} & NO & 35(58.3) & $17(56.7)$ & 0.88 \\
\hline & $\mathbf{N}+$ & $25(41.7)$ & 13(43.3) & \\
\hline \multirow[t]{2}{*}{ Hypertension, n (\%) } & Yes & 17(28.3) & $8(26.7)$ & 0.868 \\
\hline & No & $43(71.7)$ & 22(73.3) & \\
\hline \multirow[t]{2}{*}{ Family history, $\mathrm{n}(\%)$} & Yes & $0(0.0)$ & $0(0.0)$ & 1.000 \\
\hline & No & $60(100.0)$ & $30(100.0)$ & \\
\hline \multirow[t]{2}{*}{ Diabetes mellitus, n (\%) } & Yes & $7(11.7)$ & $7(23.3)$ & 0.150 \\
\hline & No & 53(88.3) & $23(76.7)$ & \\
\hline \multirow[t]{2}{*}{ Smoke, n (\%) } & Yes & 18(30.0) & $9(30.0)$ & 1.000 \\
\hline & No & 42(70.0) & $21(70.0)$ & \\
\hline \multirow[t]{2}{*}{ Drink, n (\%) } & Yes & 11(18.3) & $6(20.0)$ & 0.849 \\
\hline & No & 49(81.7) & 24(80.0) & \\
\hline
\end{tabular}




\section{ADC} Inertia_angle 135_offset7
unitormity Uniformity
InverseDifferenceMoment_AlDirection_offset7
GLCMEnergy_angle45_offset7 LongRunLowGreyleve IEIEmphasis_angleo_offset? RelativeDeviation

ClusterProminence_AlDirection_offset4_SD Correlation_angle90_offset1 GreyLevelNonuniformity_ANDirection_offset7_SD GLCMEntropy_AlDirection_offset7_SD Inertia_angle90_offset7 Maximum3DDiameter VolumeCC GLCMEntropy_angle0_offset Sphericaloisproportion verseDifferenceMoment_angle90_offset1 RunLengthnNonuniformity_AllDirection_offset7_SD Low GreyLevelRunEmphasis All irection oftset1 GLCMEntropy_angle45_offset7 GLCMEntropy_angle0_offset? VolumeMM

GreyLevelNonuniformity_AIIDirection_offset1_SD nertia_angle 45_offset4

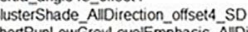
verseDifferenceMoment_angle 45_offset1 GLCMEntropy_angle90_offset7

\section{T2WI}

LongRunLowGreyLevelEmphasis_angle135_offset7 LongRunEmphasis_angle 135_offset :

Emphasis_AlDirection_oftset7 ShortRunEmphasis_Aidirection_offset Longunteris LongRunLowGreyL évelEmphasis_AllDirection offset LongRunLowGreyLevelEmphasis_angle135_offset4 LongRunEmphasis_angle 90 offset 1 LongRunLowGreyLevelEmphasis_angle90_offset7 LongRunLowGreyLevelemphasis_angle45_offset7 LongRunLowGreyLevelEmphasis AlDirection oftset InverseDifference Moment_AIDirection offset7 7 SD ShortRunEmphasis_angleo_offset1 ShortRunEmphasis_angle45_offset ClusterShade_Alioirection_offset1 LowGreyLevelRunEmphasis_angle 135_offset MeanDeviation

LongRunEmphasis Aldirection offset7 SD LongRunHighGreyLevelEmphasis _AlDirection offset1 SD stdDeviation

Variance
LongRunLowGreyLevelEmphasis_angle0_offset4 LongRunEmphasis_angleo_offset7

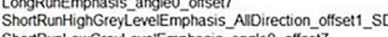
ShortRunLowGreyLevelEmphasis_angle0_offset7
ClusterShade_angle 135 offset1

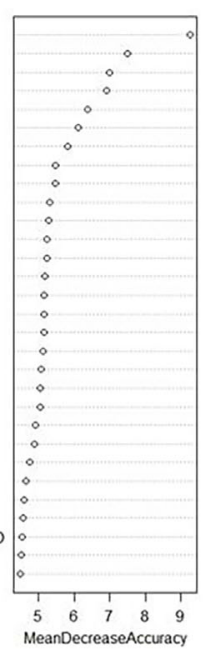

Inertia_angle135_offset7

uniformity

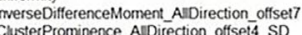

GLCMEntropy CA_-Aidirection_offsel4_SD

LowGreyLevelRunEmphasis_AlDirection_offset1_SD

GreyLevelNonuniformity_AIDirection_oftset7_SD

GLCMEntropy_angle45_offset?

VolumeCC

GLCMEnergy_angle45_offset?

GLCMEntropy_angle0_offset1

Compactness
Volumelum

Imversedifferencemoment_angle 135_offset7

inertia_Alidirection_offset4_SD

verse Difference Moment_angle 135 _offset 1

LongRunLowGreyLevelEmphasis_angleo_offset7

Sphericali Disproportion

GLCMEntopy_Aidirection_offset

SurfaceArea Maximum3DDiameter

Iverse DifferenceMoment_angle90_offset

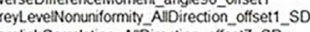

GLCMEntropy_angleo offset?

GLCMEnergy_AlDitrection_offset7

GLCMEntropy_angle135_offset1

MeanDecreaseAccurac

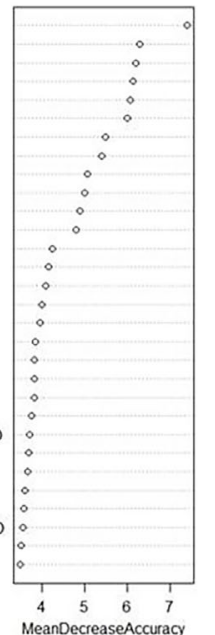

LongRunLowGreyLevelEmphasis_angle 135_offset7 ongRunLowGreyLevelEmphasis_AilDirection_offset? LongRunEmphasis_AlDirection_offset LhortRunEmphasis_angle 135_offset1 ShortRunEmphasis AllDirection offset1 LongRunLowGreyLevelEmphasis_angle 135_offset4 LongRunEmphasis_angle 135_oftset1 LongRunLowGreyLevelEmphasis_angle90_offset7 LongRunLowGreyLevelEmphasis_angle45_offset7 ongRunEmphasis_anglego_otiset1

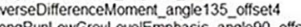

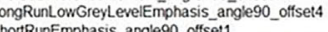
ImerseDifferencemoment_Aildirection_offset7_SD LongRunL owGreyLevelEmphasis_AlDirection_offset LowGreyLevelRunEmphasis_angle 135_offset 1 ongRunEmphasis_Airitireciron_ofiset7_SD

LowGreyLevelRunEmphasis_angle90_offset1 Vanance

OngRunLowGreyLevelEmphasis angleo offse: LoangRunLowGreyLevelEmphasis_angle90_offset1 ShortRunEmphasis_Ailirection_offset1_SD LongRunEmphasis_AiDitection_offset__SD

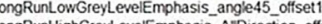
LongRunHighGreyLevelEmphasis_AiDirection
LowGreyLevelRunEmphasis_angle45_offset4
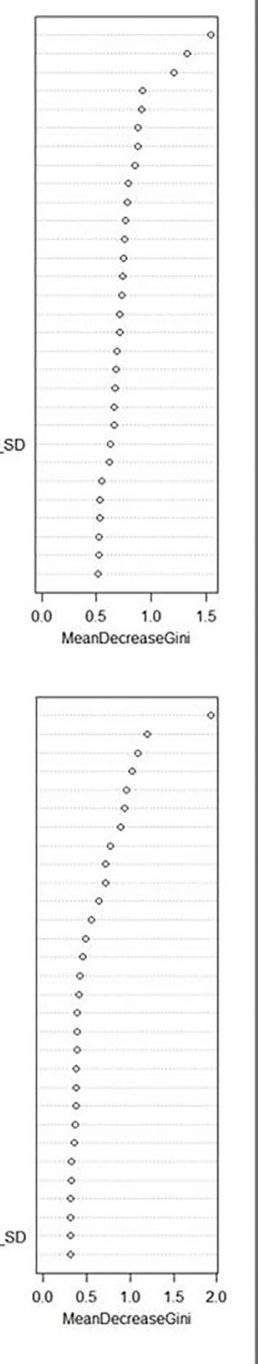

FIGURE 5 | The importance rank of features in T2W and ADC images.

each patient via a linear combination of the selected features that were weighted by their respective coefficients. Combined score was calculated via a linear combination of the ADC score and T2 score. The calculation formula was as follows:

T2 Score $=773+991 *$

LongRunLowGreyLevelEmphasis _AllDirection_offset7 - 777*

ShortRunEmphasis_angle135_offset1 .

ADC Score $=-5.48+1.4 * 10^{-3} *$

RelativeDeviation $+8.33 *$ uniformity +0.72

*LongRunLowGreyLevelEmphasis_angle0_offset7 .
Combine Score $=0.092+1.17 \star \mathrm{T} 2$ Score $+0.92 \star \mathrm{ADC}$ Score.

\section{Performance of the Radiomics Models}

The established T2 score, ADC score, and combined score were significantly associated with the status of MSI in both training and testing sets. The scores were all significantly higher in MSI group than that in MSS group for both training and testing sets. The box plot was shown in Figure 6. The performance was better for combined score with an AUC of 0.908 (95\% CI, 0.845-0.971) in training set and 0.926 (95\% CI, 0.813-1.0) in testing set than either one, where T2 score showed an AUC of 0.870 (95\% CI: 0.794-0.945) in training set and 0.895 (95\% CI: 0.777-1.000) in testing set and the ADC score showed an AUC of $0.790(95 \% \mathrm{CI}$ : 


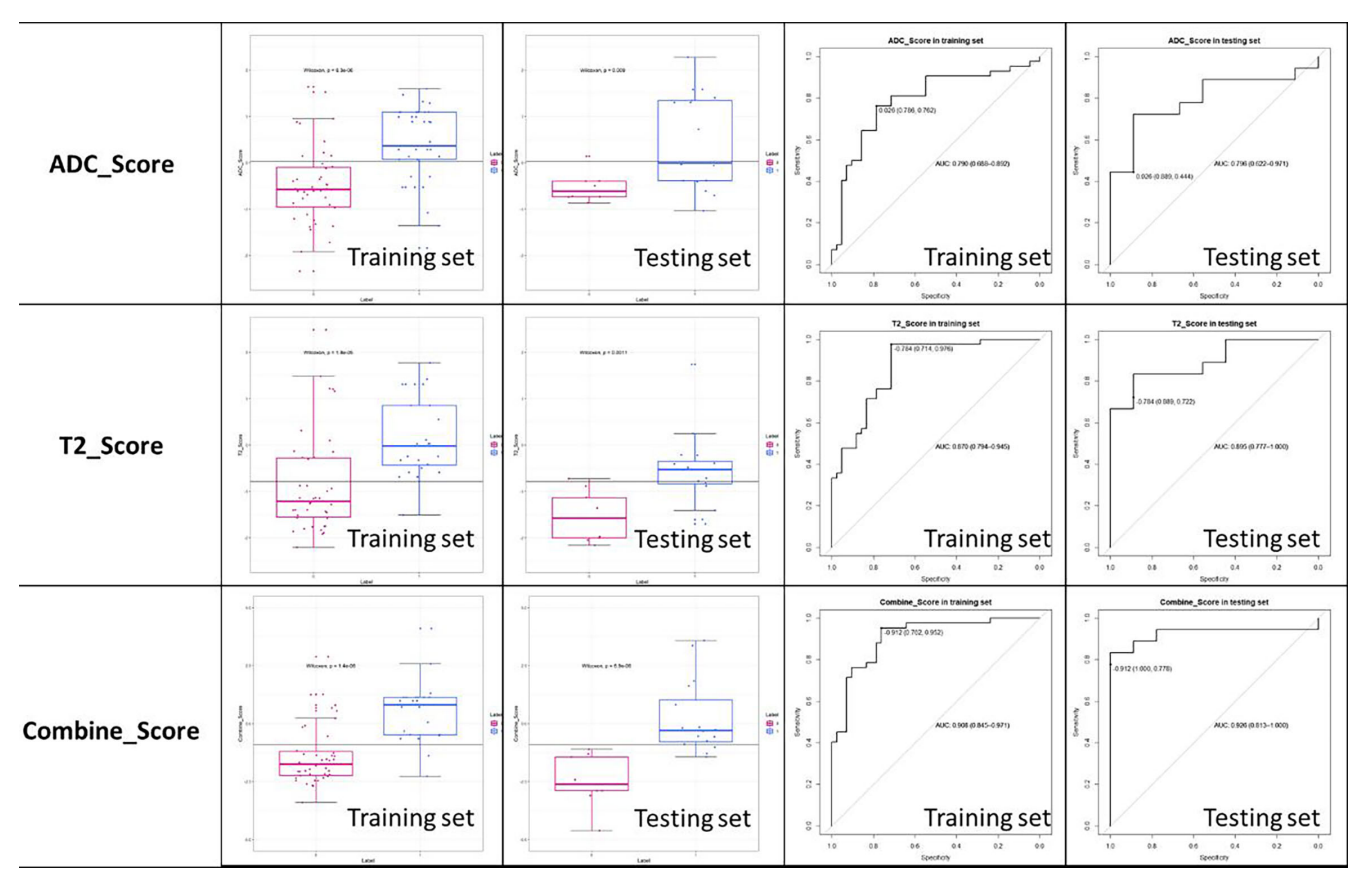

FIGURE 6 | The box-plot and ROC curve of ADC score, T2 score, and combined score.

0.794-0.945) in training set and 0.796 (95\% CI: 0.622-0.971) in testing set. The accuracy, sensitivity, and specificity based on the optimal cutoff value for all the models were shown in Table 2 and Figure 6. Delong test demonstrated that there was no significant difference between the performance of the model in training and testing set, which showed the relative robustness of the model. The calibration curve showed that the combined model fitted well, and the decision curve analysis showed the clinical usefulness of the combined model, as shown in Figure 7.

\section{DISCUSSION}

Our study revealed that the models of radiomics features based on T2W and ADC imaging can preoperatively predict the MSI status efficiently. The prediction performance of combined score (T2 and $\mathrm{ADC}$ ) has a higher degree of specificity than T2 score or ADC score.

CT can also be used as a technique to determine MSI status, all the measurements (NICA, V, D; kP, A, V, D; Eff-Z) of MSI based on energy spectrum CT were significantly lower, where MSS and AUC of multivariate logistic regression analysis was 0.886 (13). In our research, the combined score with an AUC of 0.908 (95\% CI: 0.845-0.971) in the training set and $0.926(95 \%$ CI: $0.813-1.0)$ in the testing set, which was better than CT. The reason might be that different sequences of $\mathrm{MR}$ represent the signal strength and quantitative parameters and could reflect heterogeneity of rectal cancer. Moreover, because of the presence of radiation in CT examination, patients with colorectal cancer who received neoadjuvant therapy and underwent multiple reexaminations may receive more radiation dose, which could become a factor in influencing the outcome.

Different tumor histology showed different characteristics of radiomics based on tumor images, the difference in texture and histogram between MSI and MSS of rectal cancer indicated the different grayscale distribution of the two images. The histogram feature described the overall distribution of grayscale in the ROI of image (14) and the texture feature described the distribution of local grayscale values of the image (15). ADC images could reflect the

TABLE 2 | Performance of the Rad-Scores established based on T2W, ADC Images and both combination for MSI and MSS Discrimination.

\begin{tabular}{|c|c|c|c|c|c|c|c|c|}
\hline Sequence & Data Set & AUC & $95 \% \mathrm{Cl}$ & ACC & Specificity & Sensitivity & Cutoff & $\begin{array}{c}\text { P values } \\
\text { (DeLong test) }\end{array}$ \\
\hline T2WI & Train & 0.870 & $0.794-0.945$ & 0.845 & 0.714 & 0.976 & 0.314 & 0.724 \\
\hline \multirow[t]{2}{*}{ ADC } & Train & 0.790 & $0.794-0.945$ & 0.774 & 0.714 & 0.976 & 0.507 & 0.949 \\
\hline & Test & 0.858 & $0.738-0.978$ & 0.722 & 0.889 & 0.556 & 0.507 & \\
\hline \multirow[t]{2}{*}{ T2WI+ADC } & Train & 0.908 & $0.845-0.971$ & 0.857 & 0.762 & 0.952 & 0.290 & 0.782 \\
\hline & Test & 0.926 & $0.813-1.000$ & 0.852 & 1.000 & 0.778 & 0.290 & \\
\hline
\end{tabular}



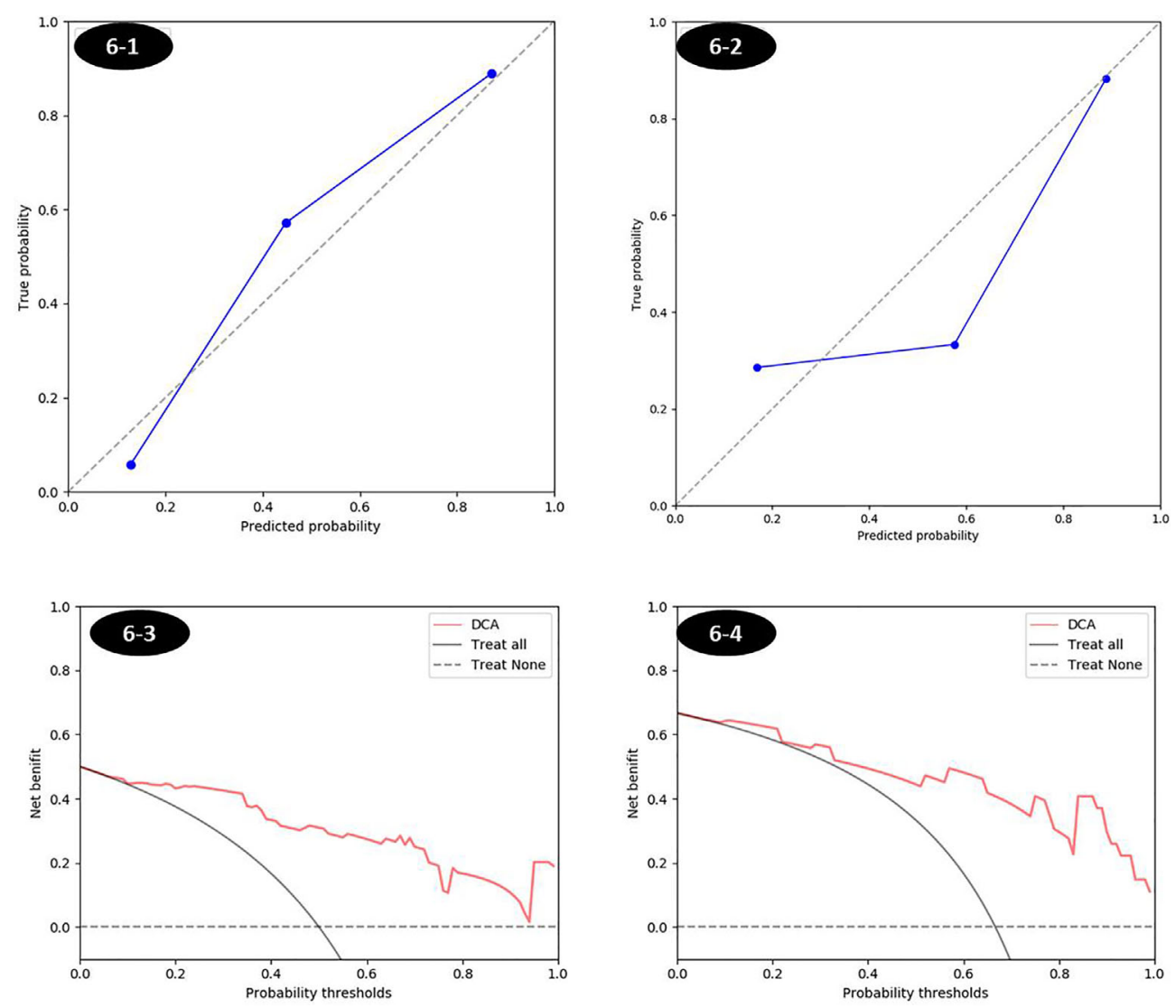

FIGURE 7 | The calibration curves and decision curves of combined score in training and testing sets. (1) Calibration curve was shown in Figures 6-1 and 6-2, respectively, for training and testing sets. The closer to the diagonal reference line, the better the fitness of the model. (2) The decision curve in Figures 6-3 and 6-4 was for the combined score in training and testing set respectively, which showed that whatever the threshold probability was, using the combined score to predict the MSI status added more benefit than either the treat-all scheme (assuming all lesions are MSI) or the treat-none scheme (assuming all lesions are MSS).

degree of diffusion of extracellular water molecules and the ADC value of malignant tumors was significantly higher than that of benign tumors, which was due to the rapid growth of malignant tumor cells, small extracellular space, and limited movement of water molecules (16). Because of the loss of mismatch repair protein gene (17), tumor cells showed relative poor differentiation and relatively more mucinous tissue and necrotic components exist (18), more CD3+, CD4+, CD8+, CD20+, and CD68+ cell infiltration and showed higher microvessel density (MVD) (19), resulting in different image grayscale distribution, which caused differences in image histogram features and texture features. Therefore, they could reflect certain spatial characteristics and could predict the MSI status. Meanwhile, T2W images formed by the signal intensity were susceptible to the MR field strength, excitation times, and noise. ADC image consists of ADC values at different locations in the image. The different histological states in the tissues and cells of colorectal cancer may lead to different extracellular space and imaging signal intensity, resulting in the combination of T2 score and ADC score, which had a better prediction effect than T2W Score or ADC image score alone.

To avoid the influence of the difference between the scanning parameters of $\mathrm{t} 2 \mathrm{w}$ and ADC images, we performed image histogram matching and homogenization processing first to make the contrast of each image consistent, ensuring that the extracted features were credible before delineating the ROI. Second, we performed a consistency test on the features extracted by two radiologists and retained the features with a consistency coefficient of $>0.75$ to reduce the error caused by the physician manually delineating. In our study, because of the presence of 30 MSI patients and 60 MSS patients, there was an inter-group data imbalance. Therefore, we used the SMOTE method to strike a balance with the MSI oversampling. At the same time, we also calculated the results without amplification in the MSI group, suggesting that the T2W and ADC model significantly improved after using the SMOTE method. This shows that the performance of the radiomics model can be further improved by using the balanced data set.

Our research currently had some limitations. First, only 90 patients were enrolled, and the number of patients needed to be increased. Second, our study used immunohistochemistry to detect the expression of mismatch repair proteins to determine the MSI status of rectal cancer; however, a study has shown that some colorectal cancers may lose MSH6 gene inactivation after receiving neoadjuvant therapy, and resulting in the loss of MSH6 
protein expression, which would cause a certain degree of inaccurate in immunohistochemistry results (20). This part of the patients had better undergo PCR to determine the true microsatellite status. In our study, there were two patients with MSH6 deletion, but no PCR test was conducted, which may affect the experimental results.

\section{DATA AVAILABILITY STATEMENT}

The raw data supporting the conclusions of this article will be made available by the authors, without undue reservation.

\section{ETHICS STATEMENT}

The studies involving human participants were reviewed and approved by The Institutional Ethical Committee of Jilin University. Written informed consent for participation was not required for this study in accordance with the national legislation and the institutional requirements. The animal study was reviewed and approved by The Institutional Ethical Committee of Jinlin University.

\section{REFERENCES}

1. Li D, Li Q, He Y. Epidemiology of Colorectal Cancer. Cancer Prev Treat (2015) 42(03):305-10. doi: 10.3971/j.issn.1000-8578

2. Ribic CM, Sargent DJ, Moore MJ, Thibodeau SN, French AJ, Goldberg RM, et al. Tumor Microsatellite-Instability Status as a Predictor of Benefit From Fluorouracil-Based Adjuvant Chemotherapy for Colon Cancer. N Engl J Med (2003) 349(3):247-57. doi: 10.1056/NEJMoa022289

3. Popat S, Hubner R, Houlston RS. Systematic Review of Microsatellite Instability and Colorectal Cancer Prognosis. J Clin Oncol (2005) 23(3):60918. doi: 10.1200/JCO.2005.01.086

4. Robert G, Hyeja K, Hsieh ET, Aronson MD, Holowaty EJ, Bull SB, et al. Tumor Microsatellite Instability and Clinical Outcome in Young Patients With Colorectal Cancer. N Engl J Med (2000) 342(2):69-77. doi: 10.1056/ NEJM200001133420201

5. Sargent DJ, Silvia M, Genevieve M, Thibodeau SN, Roberto L, Hamilton SR, et al. Defective Mismatch Repair as a Predictive Marker for Lack of Efficacy of Fluorouracil-Based Adjuvant Therapy in Colon Cancer. J Clin Oncol (2010) 28(20):3219-26. doi: 10.1200/JCO.2009.27.1825

6. Du C, Zhao J, Xue W, Dou F, Gu J. Prognostic Value of Microsatellite Instability in Sporadic Locally Advanced Rectal Cancer Following Neoadjuvant Radiotherapy. Histopathology (2013) 62(5):723-30. doi: 10.1111/his.12069

7. Demes M, Scheil-Bertram S, Bartsch H, Fisseler-Eckhoff A. Signature of Microsatellite Instability, KRAS and BRAF Gene Mutations in German Patients With Locally Advanced Rectal Adenocarcinoma Before and After Neoadjuvant 5-FU Radiochemotherapy. J Gastrointestinal Oncol (2013) 4 (2):182-92. doi: 10.3978/j.issn.2078-6891.2013.012

8. Mike N, Sam TS, Marissa L, Elizabeth L, Amy D, Hans P, et al. An Update on the Use of Immunotherapy in Patients With Colorectal Cancer. Expert Rev Gastroenterol Hepatol (2021) 15(3):291-304. doi: 10.1080/17474124.2021.1845141

9. Gado A, Ebeid B, Abdelmohsen A, Axon A. Improving the Yield of Histological Sampling in Patients With Suspected Colorectal Cancer During Colonoscopy by Introducing a Colonoscopy Quality Assurance Program. Gastroenterol Res (2011) 4(4):157-61. doi: 10.4021/gr334w

10. Yang L, Dong D, Fang M, Zhu Y, Zang Y, Liu Z, et al. Can CT-Based Radiomics Signature Predict KRAS/NRAS/BRAF Mutations in Colorectal Cancer? Eur Radiol (2018) 28(5):2058-67. doi: 10.1007/s00330-017-5146-8

11. Li H, Zhu Y, Burnside E, Huang E, Drukker K, Hoadley K, et al. Quantitative MRI Radiomics in the Prediction of Molecular Classifications of Breast Cancer Subtypes in the TCGA/TCIA Data Set. NPJ Breast Cancer (2016) 2 (1):9-29. doi: 10.1038/npjbcancer.2016.12

\section{AUTHOR CONTRIBUTIONS}

ZL: methodology, resources, and data curation. HD: methodology, conceptualization, and investigation. YL: conceptualization and supervision. FP: writing - original draft and data curation. YY: resources and data curation. MZ: project administration, supervision, and funding acquisition. All authors contributed to the article and approved the submitted version.

\section{FUNDING}

This study was financially supported by the key project grant from the Natural Science Foundation of Jilin Province, China (No. 20180101125JC), the Key Project of Social Development Fund of Science and Technology Department of Jilin Province, China (No. 20200403129SF), the National Natural Science Foundation of China, 81971573 to HD, the Suzhou Gusu Medical Youth Talent, GSWS2020019 to HD, and the 13th Five-Year Science and Technology Project of the Education Department of Jilin Province, JJKH20190064KJ to MZ.

12. Fetit A, Novak J, Rodriguez D, Auer D, Clark C, Grundy R, et al. Radiomics in Paediatric Neuro-Oncology: A Multicentre Study on MRI Texture Analysis. NMR Biomed (2018) 31(1):3781. doi: 10.1002/nbm.3781

13. Wu J, Lv Y, Wang N, Zhao Y, Zhang P, Liu Y, et al. The Value of Single-Source Dual-Energy CT Imaging for Discriminating Microsatellite Instability From Microsatellite Stability Human Colorectal Cancer. Eur Radiol (2019) 29 (7):3782-90. doi: 10.1007/s00330-019-06144-5

14. Just N. Improving Tumour Heterogeneity MRI Assessment With Histograms. Br J Cancer (2014) 111(12):2205-13. doi: 10.1038/bjc.2014.512

15. Guan Y, Li W, Jiang Z, Zhang B, Chen Y, Huang X, et al. Value of WholeLesion Apparent Diffusion Coefficient (ADC) First-Order Statistics and Texture Features in Clinical Staging of Cervical Cancers. Clin Radiol (2017) 72(11):951-8. doi: 10.1016/j.crad.2017.06.115

16. Song Y, Yoon Y, Chong Y, Seo S, Choi Y, Sohn I, et al. Diagnostic Performance of Conventional MRI Parameters and Apparent Diffusion Coefficient Values in Differentiating Between Benign and Malignant Soft-Tissue Tumours. Clin Radiol (2017) 72(8):691.e1-.e10. doi: 10.1016/j.crad.2017.02.003

17. Boland C, Goel A. Microsatellite Instability in Colorectal Cancer. Gastroenterology (2010) 138(6):2073-87.e3. doi: 10.1053/j.gastro.2009.12.064

18. Gupta R, Sinha S, Paul R. The Impact of Microsatellite Stability Status in Colorectal Cancer. Curr Prob Cancer (2018) 42(6):548-59. doi: 10.1016/ j.currproblcancer.2018.06.010

19. De Smedt L, Lemahieu J, Palmans S, Govaere O, Tousseyn T, Van Cutsem E, et al. Microsatellite Instable vs Stable Colon Carcinomas: Analysis of Tumour Heterogeneity, Inflammation and Angiogenesis. Br J Cancer (2015) 113 (3):500-9. doi: 10.1038/bjc.2015.213

20. Kuan S, Ren B, Brand R, Dudley B, Pai R. Neoadjuvant Therapy in Microsatellite-Stable Colorectal Carcinoma Induces Concomitant Loss of MSH6 and Ki-67 Expression. Hum Pathol (2017) 63:33-9. doi: 10.1016/ j.humpath.2017.02.003

Conflict of Interest: The authors declare that the research was conducted in the absence of any commercial or financial relationships that could be construed as a potential conflict of interest.

Copyright (c) 2021 Li, Dai, Liu, Pan, Yang and Zhang. This is an open-access article distributed under the terms of the Creative Commons Attribution License (CC BY). The use, distribution or reproduction in other forums is permitted, provided the original author(s) and the copyright owner(s) are credited and that the original publication in this journal is cited, in accordance with accepted academic practice. No use, distribution or reproduction is permitted which does not comply with these terms. 